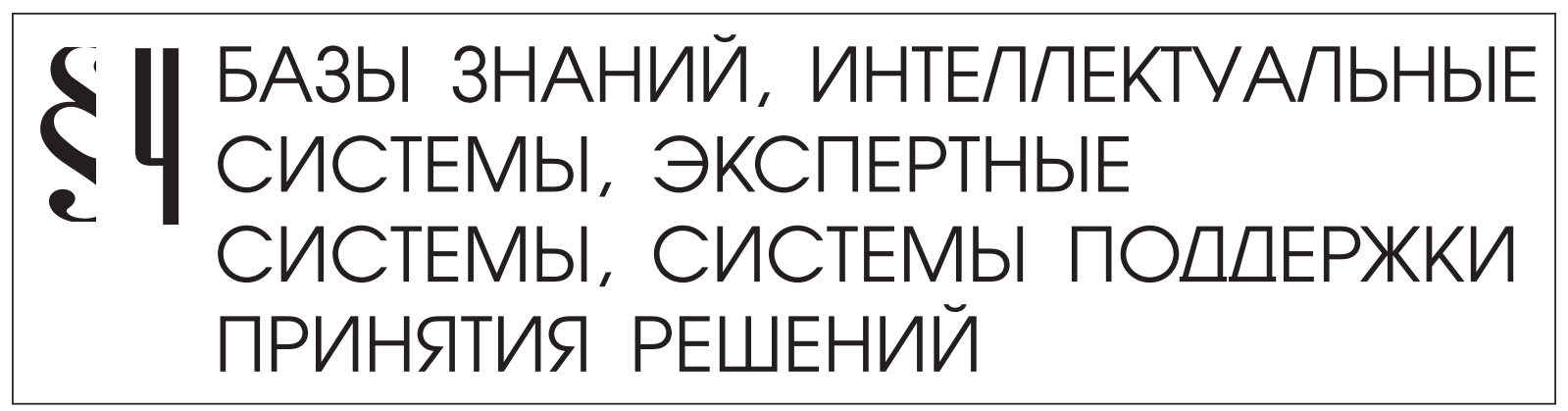

Бородин А. В.

\title{
АРХИТЕКТУРА ИНФОРМАЦИОННОЙ СИСТЕМЫ ПОДДЕРЖКИ ПРИНЯТИЯ РЕШЕНИЙ ПО УПРАВЛЕНИЮ ПЕРСОНАЛОМ РОЗНИЧНОЙ ПОДСИСТЕМЫ КОММЕРЧЕСКОГО БАНКА
}

\begin{abstract}
Аннотация: Вусловиях сокращения объемов корпоративного рынка для многих филиалов коммерческих банков чентр внимания фронт-офисных подразделений все чаще перемещается в сторону розничного сегмента. Однако, огромная конкуренция в розницезаставляет банки двигаться в сторону непопулярных мер по интенсификации труда и "оптимизации персонала". В этих условиях традиционные методы принятия решений, характерные для кадровых подразделений, работают плохо. Требуются новые подходы к извлечению данных для принятия решений, необходимы инструменты объективного анализа ситуации и выработки оптимальных решений.Описанию варианта практического решения указанных проблем посвящена настоящая работа. Иначе говоря, предметом исследования здесь является HR-прочесс в розничной подсистеме коммерческого банка. Исследование процессов принятия решений в HR-подсистеме коммерческого банка проведено с позиций системного анализа. Разработана визуальная модель прочессов, характерных для подсистемы, выявлены источники информации для принятия решений. Далее, используя построенную модель, на основе методов имитационного моделирования и численной оnтимизации разработана технология автоматизированной подготовки рекомендаций по кадровой политике розничных подсистем кредитныхучреждений. Подход, предложенный в данной работе, принципиально отличается от аналогов широтой охвата доступных источников информации и методами извлечения знаний из баз данных. Предложенный подход впервые реализует метод имитационного моделирования операционных рисков розничной подсистемы банка, позволяющий построить полное вероятностное пространство исходов. Тем самым обеспечивается принципиально более высокая точность и устойчивость расчетов нежели при использовании методов Монте-Карло при сравнимых вычислительных затратах.
\end{abstract}


Ключевые слова: вычислительная сложность, имитационное моделирование, сети Петри, риск, поддержка принятия решений, управление персоналом, коммерческий банк, класс сложности, оптимизация, метод Нелдера-Мида

\section{Введение.}

Многие коммерческие банки (КБ) современной России активно развивают розничный сегмент своего бизнеса. Для одних - это значимый источник ресурсов. Для других - сфера специализации в части продвижения ссудного продукта. Однако для обеих групп банков подбор фронт-офисного персонала оказывается «головной болью». Действительно, с одной стороны, общее требование повышения эффективности банковской деятельности определяет, во-первых, необходимость внедрения технологий интенсификации труда, во-вторых, необходимость использования различных систем мотивации персонала, и, все это, на фоне жесткого лимитирования фонда оплаты труда (ФОТ). С другой стороны, качество персонала зависит от его способности к обучению, ответственности сотрудников за результаты своего труда, наличия достаточного опыта работы и стремления к личному росту. Перечисленные требования и личные качества сотрудников во многом противоречат друг другу. Например, интенсификация труда, опыт персонала и сепаратная мотивация вступают в противоречие с ограничением ФОТ. Заметная способность к обучению и невысокая требовательность к уровню оплаты труда (со стороны молодых специалистов) часто не способствует повышению ответственности. Кроме того способности к эффективному обучению на фоне спорной общей мотивации значительно повышают риск мошенничества со стороны сотрудников. Таким образом, персонал розничного подразделения КБ - источник ошибок и, следовательно, убытков. В этих условиях задача эффективного управления персоналом становится для КБ крайне трудной и чрезвычайно актуальной $[1,8,16]$.

\section{Цель исследования.}

Данная работа посвящена разработке подхода куправлению персоналом в розничной подсистеме КБ на основе методов теории риска и формированию архитектуры соответствующей информационной системы. В качестве ожидаемого результата исследования предполагается создание инструмента выбора оптимальной политики мотивации персонала по критерию совокупной доходности розничного дивизиона КБ (с учетом потерь от ошибок и мошенничества). В качестве программы исследований можно указать решение следующих задач:

1. выбор критерия оптимальности функционирования HR-процесса подразделения в условиях операционных рисков различного генезиса;

2. определение перечня источников информации для решения первой поставленной задачи;

3. декомпозиция основной задачи на подзадачи и подбор адекватных экономико- 
математических методов для их решения;

4. реализация системы экономико-математических моделей в форме соответствующего программного обеспечения;

5. решение конкретной задачи синтеза оптимальной стратегии управления персоналом розничной подсистемы банка;

6. выбор методики оценки экономической эффективности внедрения предложенных решений;

7. оценка экономической эффективности конкретной стратегии управления персоналом розничной подсистемы банка;

8. анализ перспектив внедрения конкретной стратегии управления персоналом розничной подсистемы банка в контексте общей стратегии развития банка.

\section{Источники информации.}

Анализ технологических процессов банка, выбранного в качестве полигона, позволил сформировать перечень основных баз данных (БД) - источников информации для данного исследования (см. рис. 1):

- БД аналитической подсистемы,

- БД «Кадры-Зарплата»,

- БД розничной подсистемы автоматизированной банковской системы (Retail),

- БД риск-событий.

\section{Методы исследования.}

Основой используемого в данной работе подхода является модель обслуживания одного клиента одним сотрудником розничной подсистемы КБ $[5,17]$. В качестве языка описания модели используется стохастическое расширение сетей Петри $[3,4,6,7]$. Модель представлена на рис. 2. Описания позиций и переходов модели, представленной на рисунке, приведены в таблице 1. Характеристикой переходов $t_{11}, t_{21}$ и $t_{22}$ являются соответствующие событиям средние убытки, а переходов $t_{12}$ и $t_{23}$-средняя прибыль на одну операцию.

На основе описанной модели одной операции строится модель обслуживания клиентов в течение заданного интервала времени всей розничной подсистемой КБ (см. рис. 3). Эта модель предполагает, что обслуживание клиентов осуществляют $m$ сотрудников, каждый из которых в течение заданного времени обслуживает в среднем $n$ клиентов. События, связанные с обслуживанием потока клиентов предполагаются независимыми. Переход $t_{0}^{1}$ соответствует событию начала обслуживания, его характеристика включает все затраты на обучение $\mathrm{m}$ сотрудников. Переход $t^{1}{ }_{1}$ соответствует событию завершения обслуживания в конце моделируемого периода. Переходы вида $t^{1}{ }_{i j}$ описывают действия $i$-го сотрудника между обслуживанием $j$-го и $j+1$-го своих клиентов. Подсеть $N_{1}$ представляет собой модель одной операции. 


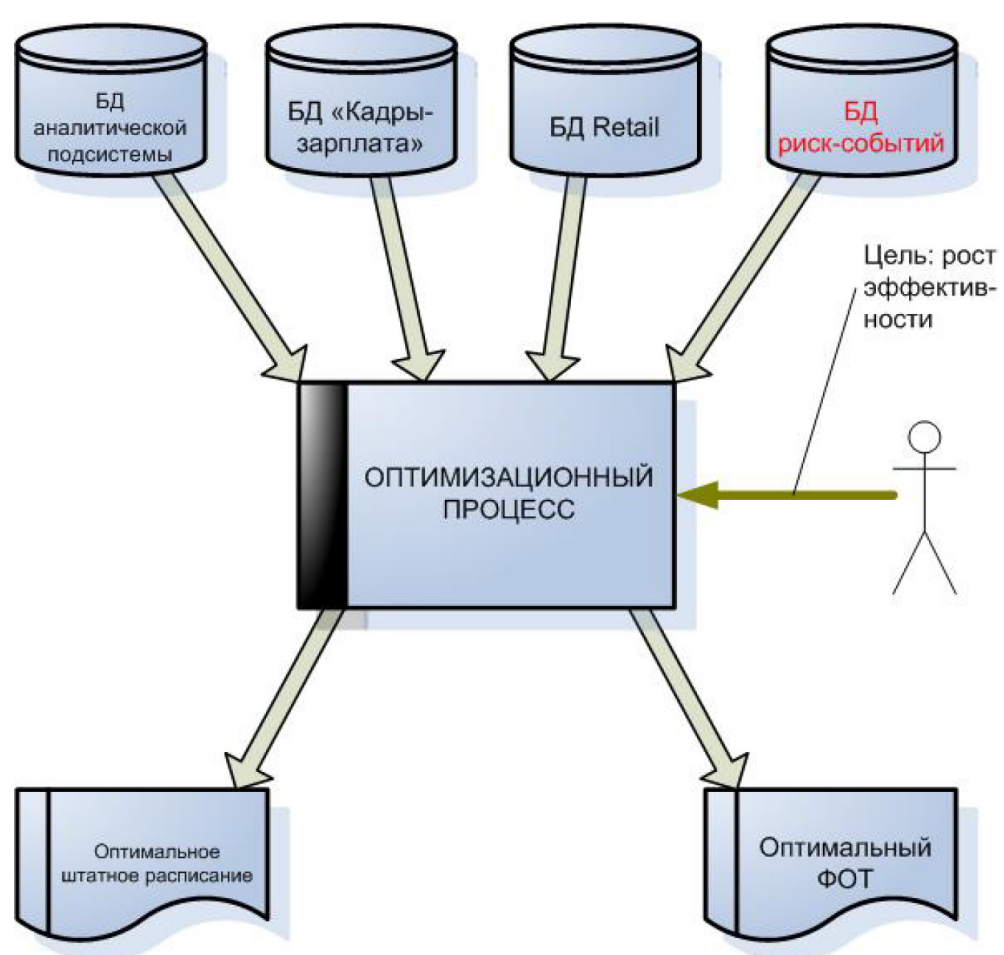

Рис. 1. Цель, источники информации и ожидаемые результаты

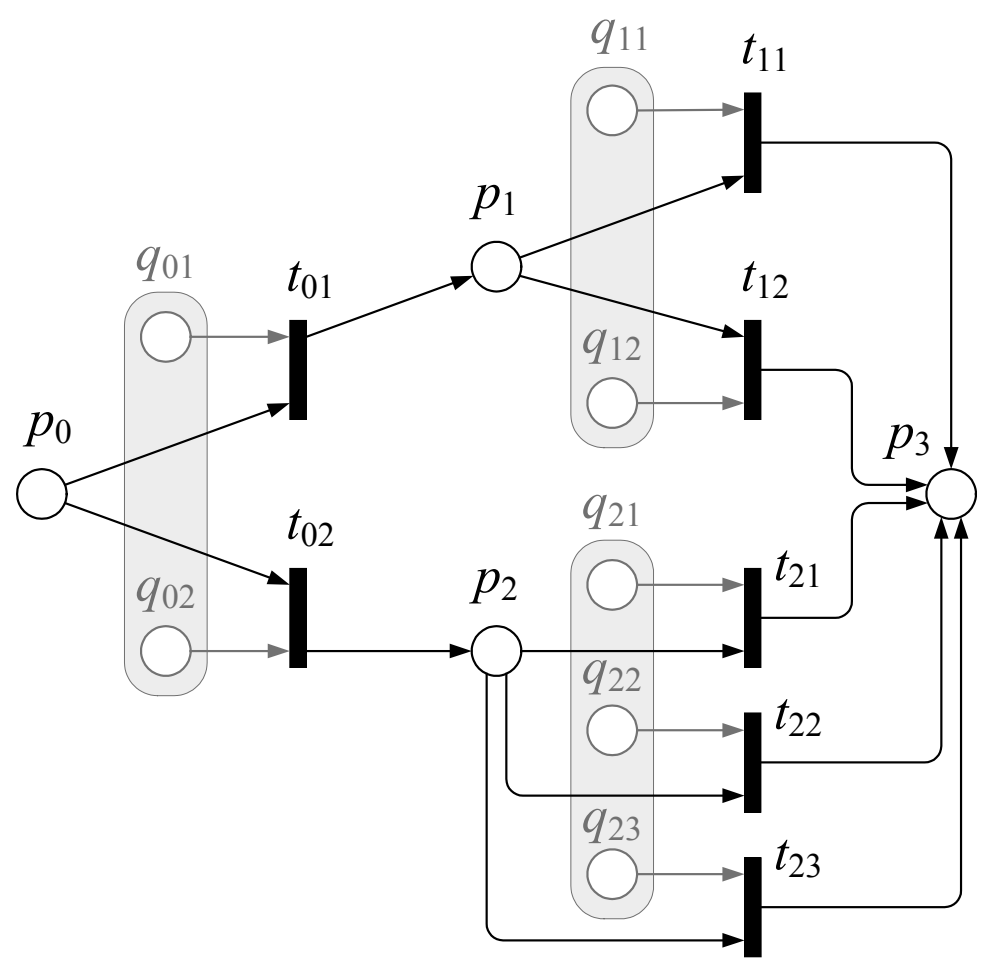

Рис. 2. Модель обслуживания клиента банка 


\begin{tabular}{|c|c|}
\hline \multicolumn{2}{|r|}{\begin{tabular}{lrl}
\multicolumn{2}{l}{} & Таблица 1 \\
\end{tabular}} \\
\hline Узел & Описание \\
\hline \multicolumn{2}{|r|}{ Стандартные позиции } \\
\hline$p_{0}$ & Условие начала обслуживания клиента банка \\
\hline$p_{1}$ & $\begin{array}{l}\text { Условие начала обслуживания клиента банка в предположении отсутствия у } \\
\text { сотрудника банка склонности к мошенничеству }\end{array}$ \\
\hline$p_{2}$ & $\begin{array}{l}\text { Условие начала обслуживания клиента банка в предположении наличия у } \\
\text { сотрудника банка склонности к мошенничеству }\end{array}$ \\
\hline$p_{3}$ & Условие завершения акта обслуживания \\
\hline \multicolumn{2}{|r|}{ Позиции первой стохастической группы } \\
\hline$q_{01}$ & $\begin{array}{l}\text { Вероятность единичной маркировки этой позиции соответствует } \\
\text { вероятности отсутствия у сотрудника склонности к мошенничеству }\end{array}$ \\
\hline$q_{02}$ & $\begin{array}{l}\text { Вероятность единичной маркировки этой позиции соответствует } \\
\text { вероятности наличия у сотрудника склонности к мошенничеству }\end{array}$ \\
\hline \multicolumn{2}{|r|}{ Позиции второй стохастической группы } \\
\hline$q_{11}$ & $\begin{array}{l}\text { Вероятность единичной маркировки этой позиции соответствует } \\
\text { вероятности совершения сотрудником ошибки }\end{array}$ \\
\hline$q_{12}$ & $\begin{array}{l}\text { Вероятность единичной маркировки этой позиции соответствует } \\
\text { вероятности безошибочной работы сотрудника }\end{array}$ \\
\hline \multicolumn{2}{|r|}{ Позиции третьей стохастической группы } \\
\hline$q_{21}$ & $\begin{array}{l}\text { Вероятность единичной маркировки этой позиции соответствует } \\
\text { вероятности совершения сотрудником ошибки }\end{array}$ \\
\hline$q_{22}$ & $\begin{array}{l}\text { Вероятность единичной маркировки этой позиции соответствует } \\
\text { вероятности возникновения благоприятных обстоятельств для } \\
\text { осуществления мошеннических действий }\end{array}$ \\
\hline$q_{23}$ & $\begin{array}{l}\text { Вероятность единичной маркировки этой позиции соответствует } \\
\text { вероятности честной безошибочной работы сотрудника }\end{array}$ \\
\hline \multicolumn{2}{|r|}{ Переходы } \\
\hline$t_{01}$ & $\begin{array}{l}\text { Переход модели в состояние отсутствия у сотрудника склонности к } \\
\text { мошенничеству }\end{array}$ \\
\hline$t_{02}$ & $\begin{array}{l}\text { Переход модели в состояние наличия у сотрудника склонности к } \\
\text { мошенничеству }\end{array}$ \\
\hline$t_{11}$ & Событие совершения ошибки при обслуживании \\
\hline$t_{12}$ & Событие безошибочного обслуживания \\
\hline$t_{21}$ & Событие совершения ошибки при обслуживании \\
\hline$t_{22}$ & $\begin{array}{l}\text { Событие совершения мошеннических действий в ходе обслуживания } \\
\text { клиента }\end{array}$ \\
\hline$t_{23}$ & Событие безошибочного обслуживания \\
\hline
\end{tabular}




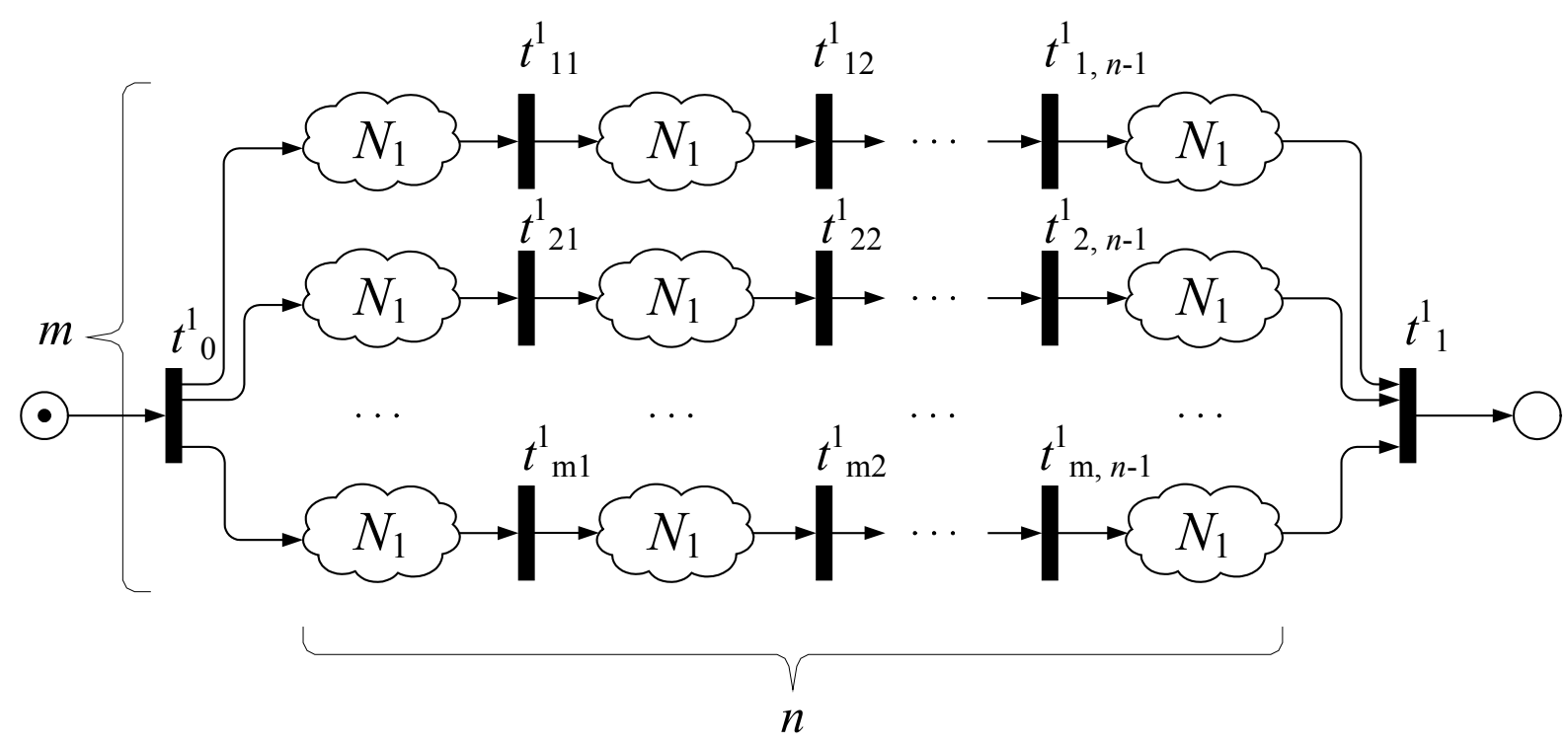

Рис. 3. Модель розничной подсистемы КБ

Пусть в модели, представленной на рис. 1, переходы $t_{11} u t_{21}$ охарактеризованы увеличением счетчика ошибок на единицу, а переход $t_{22}$ - увеличением на единицу счетчика мошенничеств. Будем использовать идеи трансляции имитационных моделей в виде сетей Петри в многочлены специального вида [14], а также алгоритмы пакета прикладных программ (ППП) «МультиМИР» [15] для расчета риска розничной подсистемы на основе полиномиального представления. В данном конкретном случае использование идей алгебры риска [15] способно очень существенно повысить эффективность вычислений ввиду однородности состава имитационной модели, приведенной на рис. 2. Используя названный инструментарий и зная вероятности единичных маркировок позиций, входящих в стохастические группы, можно рассчитать распределения случайных величин количества ошибок и количества мошенничеств в розничной подсистеме в течение заданного интервала времени. Поскольку наблюдаемые количества ошибок и мошенничеств в течение интервала времени можно рассматривать как выборочные значения из генеральных совокупностей, соответствующих вычисленным распределениям, то возможна калибровка модели. Суть калибровки заключается в подборе таких вероятностей единичных маркировок позиций, входящих в стохастические группы, которые бы максимизировали правдоподобие принадлежности наблюдаемых значений соответствующим генеральным совокупностям для ряда последовательно расположенных интервалов времени. Фактически калибровка модели означает построение двухфакторной эконометрической модели

$$
\mathbf{p}=\mathbf{p}(m, n, c)
$$

где $\mathbf{p}$ - вектор вероятностей единичных маркировок позиций, принадлежащих трем стохастическим группам сети Петри $N_{1}$, с - ФОТ. Во избежание недопонимания подчеркнем 
тот факт, что данная модель является двухфакторной. Это связано с тем, что два фактора $m$ и $n$ связаны: их произведение равно суммарному количеству операций, совершенных розничным блоком за заданный период времени.

В качестве задачи управления персоналом в розничной подсистеме КБ, позволяющей определить оптимальные значения количества персонала фронт-офиса и соответствующего объема ФОТ, в рамках данной работы предлагается использовать оптимизационную задачу вида:

$$
\underset{\substack{m n=N \\ c \in C}}{\arg \max } \mu[D(m, n, c, \mathbf{p}(m, n, c))],
$$

где $\mu$ - некоторая мера риска, описываемого случайной величиной доходности розничной подсистемы $D(m, n, c, \mathbf{p})$, рассчитываемой на базе имитационной модели, представленной на рис. 1 и 2; $N$ - прогнозируемое количество клиентов розничной подсистемы в течение планируемого периода; $C$ - ограничения на ФОТ.

\section{Выбор критерия оптимальности.}

В качестве цели банковской деятельности в современном обществе декларировано извлечение прибыли. Прибыль - это фактор привлечения и стимулирования акционеров [10], прибыль - это один из основных факторов повышения рейтинга кредитного учреждения [1]. В условиях риска, когда прибыль рассматривается как случайная величина, важно определить, какую характеристику этой случайной величины использовать в качестве критерия.

Одной из таких характеристик, используемых со времен создания теории портфеля Г. Марковица, является математическое ожидание. Несмотря на имеющиеся проблемы, эта мера риска используется до настоящего времени. Здесь и далее термин «мера риска» понимается максимально широко, а именно, как некоторая функция-индикатор отношения предпочтения на множестве исходов [15].

С использованием математического ожидания в качестве меры риска связан ряд парадоксов, самый известный из которых - «Санкт-Петербургский парадокс» [12]. С целью устранения такого рода парадоксов в прошлом столетии была предложена концепция «ожидаемой полезности». То есть речь идет о мере риска - ожидаемой полезности. Эта концепция впервые позволила учесть индивидуальные особенности лица принятия решений (ЛПР). Она позволила разрешить ряд старых парадоксов, но и породила новые. Отметим тот факт, что математическое ожидание - частный случай ожидаемой полезности, когда ЛПР нейтрален к риску на всем рассматриваемом диапазоне исходов.

Идея меры ожидаемой полезности заключается в нелинейном монотонном преобразовании носителя риска при сохранении вероятностной меры при интегрировании. Другая идея (сохранить шкалу финансового результата (носитель) и подвергнуть нелинейному монотонному преобразованию вероятностную меру), использована в мере риска возмущенной вероятности [15]. Эта мера в общем виде широко применятся в 
страховании. Частный предельный случай еe, мера риска «Value at Risk» (VaR), широко используется в финансовом менеджменте и, особенно, в банковской сфере, в некотором смысле благодаря деятельности Базельского комитета по банковскому надзору. Коротко суть этой меры заключается в следующем, $\mathrm{VaR}$ - это минимальная доходность на абсолютной шкале, которая может возникнуть для позиции с вероятностью не выше заданной. С математической точки зрения $\mathrm{VaR}_{\alpha}$ - это $\alpha$-квантиль распределения случайной величины доходности [19], где $\alpha$ - это пороговая вероятность.

Поскольку модельный банк - это акционерное общество с блокирующим участием государства, в котором отсутствуют отдельные доминирующие акционеры, то в его органах управления отсутствует локализованное ЛПР, и решения принимаются коллегиально. То есть использование концепции ожидаемой полезности в этом случае не целесообразно и следует использовать математическое ожидание, наиболее адекватно отражающее мнение большого коллегиального органа.

С другой стороны, во многих случаях возможен отрицательный финансовый результат. Для учета этого случая чрезвычайно актуальна мера риска VaR. VaR со знаком «минус», в этом случае, как раз определяет объем резерва на возможные потери в условиях операционных рисков.

Учитывая два последних тезиса, в качестве критерия оптимальности принятия решений часто используют меру риска вида

$$
E(X)+\left\{\begin{array}{c|c}
\operatorname{VaR}_{\alpha}(X) & \begin{array}{|}
\mathrm{VaR}_{\alpha}(X)<0 \\
\operatorname{VaR}_{\alpha}(X) \geq 0
\end{array}
\end{array}\right\},
$$

где $X$ - случайная величина, определяющая риск, $E$ - знак математического ожидания. Данная мера риска наиболее адекватна целям данного исследования.

\section{Методика извлечения данных из БД.}

Для определения вероятностей единичных маркировок позиций стохастических групп модели операции (рис. 2) при различных параметрах кадрового обеспечения введем следующие обозначения.

Пусть $N$ - общее количество операций в розничной подсистеме за моделируемый период; $n_{1}$ - количество ошибок персонала в течение фиксированного периода; $n_{2}-$ количество выявленных мошеннических действий в течение фиксированного периода; $n_{3}$ - количество ошибок, допущенных сотрудниками в течение фиксированного периода, до факта выявления в отношении них фактов мошеннических действий. Эти данные будут исходными. Значение $N$ может быть получено из БД розничной подсистемы, значения $n_{1}, n_{2}, n_{3}-$ из БД риск-событий (см. рис. 1).

Пусть далее: $n_{01}$ - количество операций, проведенных не склонным к мошенничеству персоналом; $n_{02}$ - количество операций, проведенных склонным к мошенничеству 
персоналом; $n_{11}$ - количество ошибок, допущенных не склонным к мошенничеству персоналом; $n_{12}$ - количество актов безошибочного обслуживания клиентов со стороны не склонного к мошенничеству персонала; $n_{21}$ - количество ошибок, допущенных персоналом, склонным к мошенничеству; $n_{22}$ - количество выявленных в течение моделируемого периода мошенничеств; $n_{23}$ - количество актов безошибочного обслуживания клиентов со стороны склонного к мошенничеству персонала.

Используя введенные обозначения, оценки вероятностей единичных маркировокпозиций стохастических групп модели операции могут быть получены следующим образом:

$$
\begin{aligned}
& p_{01}=\frac{n_{01}}{N}, p_{02}=\frac{n_{02}}{N}=1-p_{01}, p_{11}=\frac{n_{11}}{n_{01}}, p_{12}=\frac{n_{12}}{n_{01}}=1-p_{11}, \\
& p_{21}=\frac{n_{21}}{n_{02}}, p_{22}=\frac{n_{22}}{n_{02}}, p_{23}=\frac{n_{23}}{n_{02}}=1-p_{21}-p_{22} .
\end{aligned}
$$

Для нахождения $n_{01^{\prime}}, n_{02^{\prime}} n_{11^{\prime}}, n_{12^{\prime}} n_{21^{\prime}} n_{22}$ и $n_{23}$ из $N, n_{1}, n_{2}$ и $n_{3}$ выпишем сначала тривиальные соотношения:

$$
\left\{\begin{array}{l}
n_{01}+n_{02}=N \\
n_{11}+n_{12}=n_{01} \\
n_{21}+n_{22}+n_{23}=n_{02} \\
n_{11}+n_{21}=n_{1} \\
n_{21}=n_{3} \\
n_{22}=n_{2} \\
n_{12}+n_{23}=N-n_{1}-n_{2}
\end{array}\right.
$$

Заметим, ранг этой системы линейных уравнений на 1 меньше числа неизвестных, то есть система имеет бесконечное количество решений.

Для преодоления этой проблемы исключим одно зависимое уравнение и воспользуемся статистикой, используемой службой безопасности банка, которая относит определенную долю персонала к группе риска.

По другой доктрине службы безопасности будем исходить из худшего случая. Так возникает задача целочисленного программирования:

$$
n_{02} \rightarrow \max
$$

при ограничениях 


$$
\left\{\begin{array}{l}
n_{01}+n_{02}=N, \\
n_{11}+n_{12}=n_{01}, \\
n_{21}+n_{22}+n_{23}=n_{02}, \\
n_{11}+n_{21}=n_{1}, \\
n_{21}=n_{3}, \\
n_{22}=n_{2}, \\
k n_{01} \geq n_{02}, \\
n_{01}, n_{02}, n_{11}, n_{12}, n_{21}, n_{22}, n_{23} \in \mathbb{Z}_{0}^{+},
\end{array}\right.
$$

где $k$ - доля персонала, относимая службой безопасности к потенциальным злоумышленникам, $0<k<1 ; \mathbb{Z}_{0}^{+}$- множество неотрицательных целых чисел.

Эта оптимизационная задача может быть решена аналитически, но в рамках описываемой информационной системы был выбран способ решения этой задачи с помощью надстройки «Поиск решения» Microsoft Excel, исходя из потенциальной возможности усложнения модели извлечения данных и обеспечения более высокой степени универсальности решения.

Результаты массового решения описанной задачи извлечения данных для последовательности, идущих друг за другом периодов, формируют ряды данных, представляющих зависимости значений вероятностей $p_{01^{\prime}} p_{02^{\prime}} p_{11^{\prime}} p_{12^{\prime}} p_{21^{\prime}} p_{22}$ и $p_{23^{\prime}}$ полученных из $N, n_{1}$ и $n_{2^{\prime}}$ от $N, m, c$. Восстановив по этим данным множественные регрессии, можно в дальнейшем использовать их в модели одной операции при вычислении совместного риска реализации всех операций, выполняемых персоналом в течении моделируемого периода времени.

\section{Принципы имитационного моделирования HR-процесса.}

Язык визуального моделирования на основе сетей Петри имеет свои достоинства и свои недостатки. В частности у этого подхода имеется существенная проблема. В рамках подхода строится полное вероятностное пространство состояний системы на заданном временном горизонте. В результате вычислительная сложность алгоритмов интерпретации визуальных моделей оказывается таковой, что эти алгоритмы следует отнести к классу ЕХРТІМЕ. Класс сложности ЕХPТІМЕ - это множество задач, решаемых детерминированной машиной Тьюринга за время $O\left(2^{p(n)}\right)[20,21]$, где $p(n)$ - полиномиальная функция от $n$, а $n$ - в данном конкретном случае - это количество элементов системы, которым присуща вероятностная неопределенность в поведении. С другой 
стороны, особенностью систем в финансово-кредитной сфере является необходимость исследования риска в портфелях, состоящих из десятков, сотен, тысяч и даже большего количества финансовых инструментов. Здесь с ростом $n$ прямая интерпретация моделей быстро перестает работать. Следует отметить, что для альтернативных подходов, основанных на использовании метода Монте-Карло [11, 13], даже при не очень больших значениях $\mathrm{n}$ начинает быстро падать точность оценки риска. Иными словами для сохранения приемлемой точности оценки риска таких портфелей с использованием метода Монте-Карло необходимы такие вычислительные затраты, которые с ростом $n$ растут также слишком быстро [9]. Казалось бы, имеет место тупиковая ситуация: никак невозможно получить оценку риска с высокой точностью для большинства интересных случаев, таких, как, например, пакет всех операций подразделения за заданный период.

Тем не менее, в последние годы, была создана теория, получившая название «Алгебра рисков». Строгое обоснование этой теории приведено в монографии [15]. В указанной работе, кроме всего прочего, показано, что если портфель финансовых инструментов состоит из нескольких субпортфелей, содержащих большое количество однородных (одинаковых, близких по характеристикам) инструментов, и все эти инструменты не зависимы, то использование алгебраических методов может обеспечить снижение сложности алгоритмов оценки риска до сложности алгоритмов, относимых к классу PSPACE $\backslash$ NL . Класс сложности PSPACE - это множество задач, которые могут быть решены машиной Тьюринга с полиномиальным ограничением пространства [18, 20]. Класс сложности NL - это множество простых задач, решаемых недетерминированной машиной Тьюринга с использованием $O(\log n)$ памяти $[18,21]$.

Рассмотрим идею использования алгебраического подхода на примере языка описания процессов риска в виде сетей Петри со случайной маркировкой отдельных групп позиций. В монографии [4] был предложен подход, основанный на представлении процесса риска в виде комбинации подсетей специального вида (см. рис.4) и использованный в данном исследовании. Каждая такая подсеть описывает элементарное дискретное вероятностное пространство с небольшим количеством исходов, статистика по которым может быть легко накоплена на основе наблюдений (или получена экспертным путем). При этом появление маркеров в позициях $q_{1}, \ldots, q_{m}$ означает возникновение возможности наступления одного из вероятных событий, описываемых переходами $t_{1}, t_{2}, \ldots, t_{n}$. Вероятность наступления каждого из этих событий описывается вероятностью маркировки соответствующей позиции $p_{1}, p_{2}, \ldots, p_{n}$ единицей, при условии отсутствия маркеров В остальных позициях этой группы позиций. В нотации алгебраического подхода [14] на рис. 4 представлен риск $\sum_{i=1}^{n} \boldsymbol{P}_{i}$, где $\boldsymbol{P}_{i}=\left\{1 *\left(\tau\left(t_{i}\right), \operatorname{Pr}\left[M\left(p_{i}\right)=1\right]\right)\right\}-i$-ая часть риска; $\tau: T \rightarrow C$ - отображение, сопоставляющее переходу преобразование системы; $T$ - множество переходов; $C$ - множество преобразований системы; $\mathrm{Pr}$ - вероятностная мера данного элементарного вероятностного пространства; $M: P \rightarrow \mathbb{Z}_{0}^{+}$- функция маркировки; $P$ - множество позиций. 
Большинство встречающихся на практике вариантов комбинаций элементарных вероятностных пространств (институциональных структур исследуемых экономических феноменов) исчерпываются рекурсивным применением конструкций, представленных на рисунках 5, 6 и 7 [14]. На рисунках рекурсия конструкций возможна на знаке облака. При этом удобно предположить, что конструкция в облаке начинается с позиции и заканчивается позицией. Если предположить, что каждое облако описывает какой-либо риск, то каждый из рисунков в предположении завершения процессов (маркер находится в последней позиции) может быть описан формулой в терминах введенных операций над рисками: рисункам 5 и 6 соответствует соотношение $\prod_{i=1}^{n} \boldsymbol{R}_{i} ;$ рисунку $7-\sum_{i=1}^{n} \boldsymbol{P}_{i} \boldsymbol{R}_{i}$.

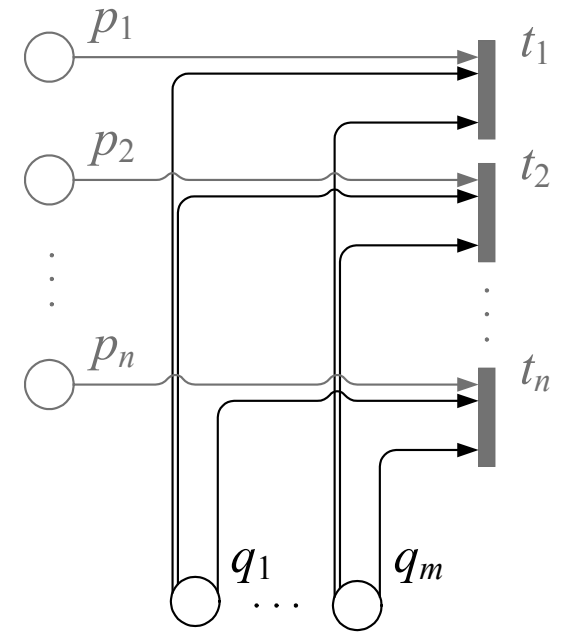

Рис. 4. Представление элементарного вероятностного пространства

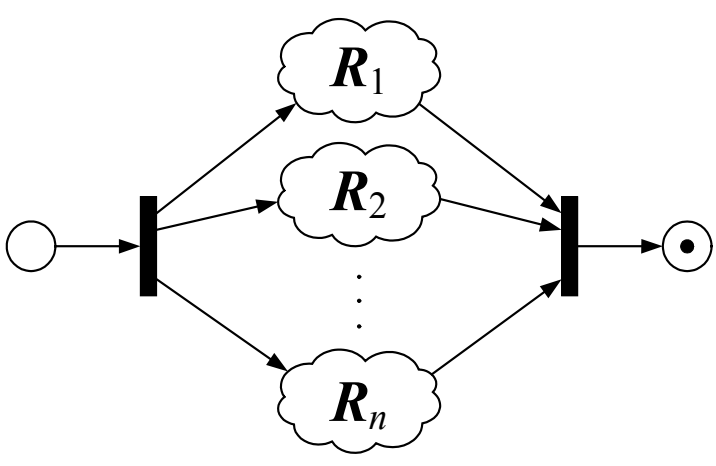

Рис. 5. Представление параллельных случайных событий

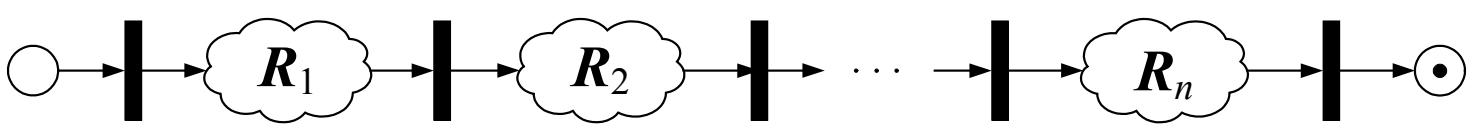

Рис. 6. Представление последовательных случайных событий 


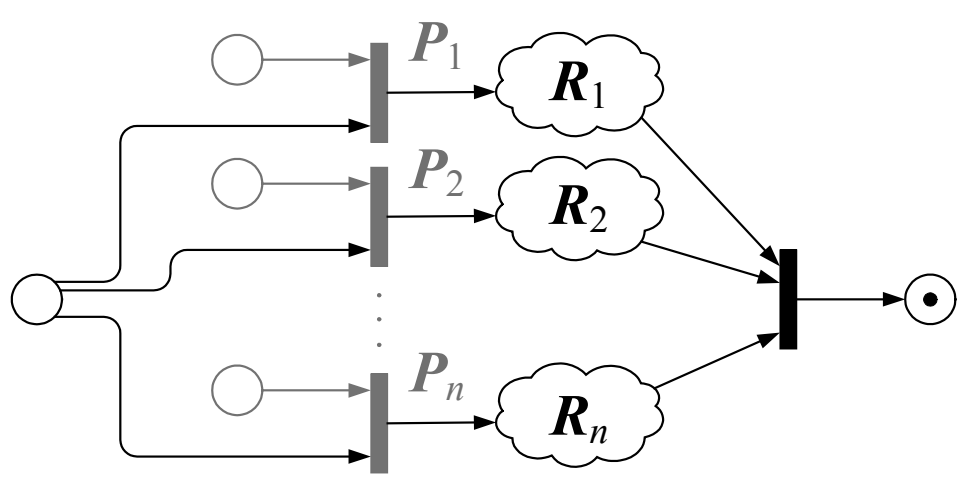

Рис. 7. Представление ветвящегося случайного процесса

Используя этот результат, можно заменить прямую интерпретацию сети Петри до заданного временного горизонта на процедуру, состоящую из двух этапов. На первом этапе подсеть Петри, ограниченная позициями, маркировка которых соответствует заданному временному горизонту, транслируется в алгебраическое выражение. На втором этапе это выражение вычисляется с использованием эффективных алгоритмов пакета прикладных программ «МультиМИР», реализующего идеи алгебры рисков [15]. Такой подход позволяет отодвинуть ограничения трудоемкости вычислений. Заметим, что речь идет о точном вычислении риска портфеля при заданной модели одного инструмента! (Когда мы говорим здесь о «точном вычислении», то понимаем это с чисто алгоритмической точки зрения и абстрагируемся от ошибок округления, накапливаемых в процессе вычислений и зависящих от используемых типов данных в соответствующей системе программирования.)

\section{Основная оптимизационная модель.}

Теперь, используя введенные обозначения, описанные принципы и критерии, можно сформулировать основную оптимизационную модель в окончательном виде:

$$
\left(m^{*}, c^{*}\right)=\underset{\substack{m n=N^{\prime} \\
c \in C}}{\arg \max }\left[E(\boldsymbol{R})+\left\{\begin{array}{c|c}
\mathrm{VaR}_{\alpha}(\boldsymbol{R}) & \mathrm{VaR}_{\alpha}(\boldsymbol{R})<0 \\
0 & \mathrm{VaR}_{\alpha}(\boldsymbol{R}) \geq 0
\end{array}\right\}\right],
$$

где $m^{*}$ - оптимальная численность персонала; $c^{*}$ - оптимальный ФОт; $N^{\prime}$ - прогнозируемое количество операций в течение модельного периода времени, для которого решается оптимизационная задача; $\boldsymbol{R}=\boldsymbol{R}_{0}{ }^{m n}$ - риск всей группы операций модельного периода; $\boldsymbol{R}_{0}$ - риск одной операции.

Для решения основной оптимизационной задачи используется метод Нелдера-Мида [2]. Этот метод не требует вычисления производных, экономичен с точки зрения количества вычислений целевой функции и неплохо справляется с негладкими функциями, к которым в общем случае относится и наша целевая функция.

В целом иерархия математических моделей, используемых в разрабатываемой ин- 
формационной системе поддержки принятия решений по управлению HR-процессом, представлена на рис. 8.

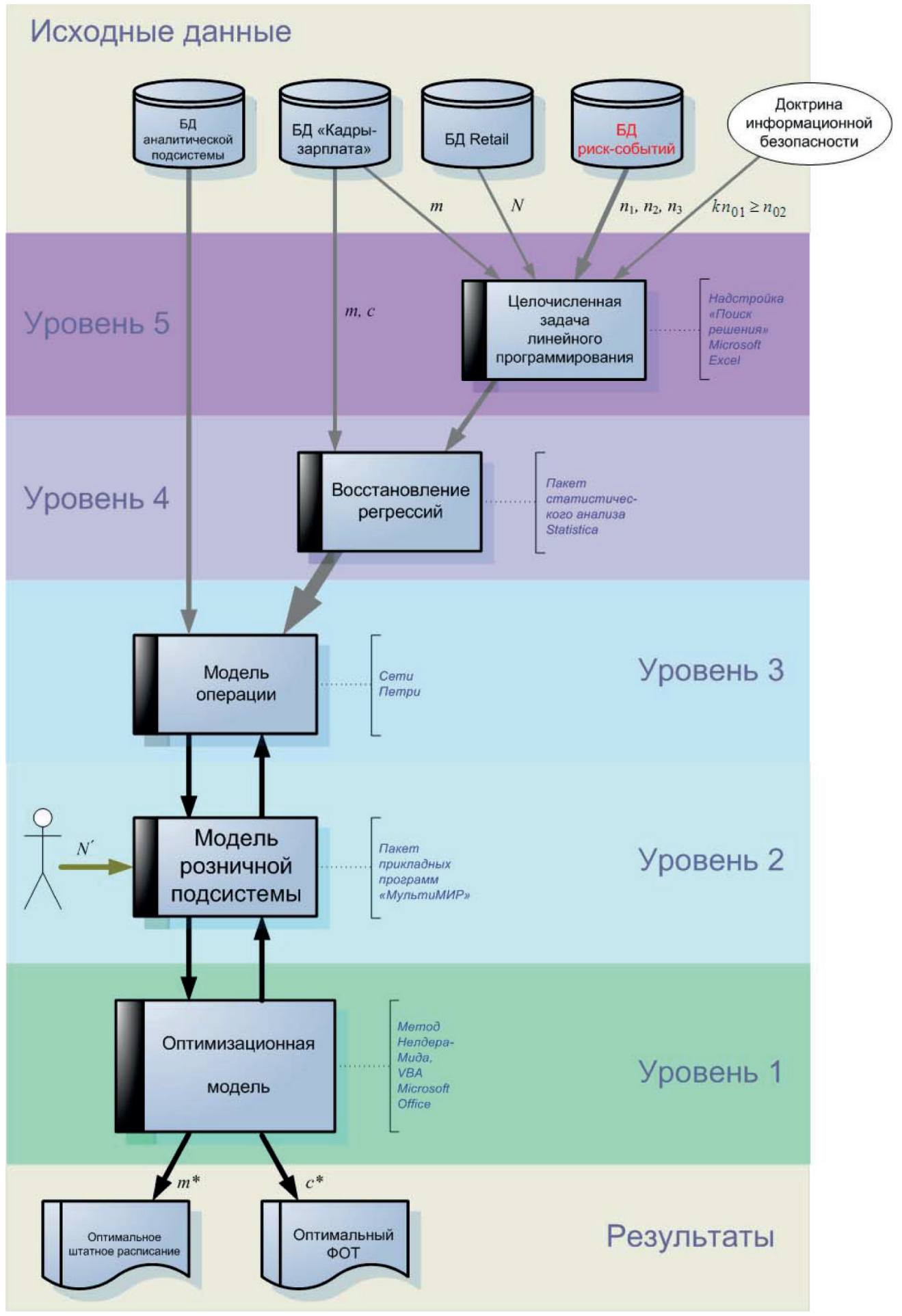

Рис. 8. Иерархия моделей управления HR-процессом 


\section{Заключение.}

Предложенная архитектура системы поддержки принятия решений использована при создании прототипа информационной системы, который в настоящее время проходит апробацию в одном из филиалов крупного российского банка. Основной целью опытной эксплуатации прототипа является отработка технологий извлечения знаний из различных БД, используемых в стандартных бизнес-процессах КБ, и проверка адекватности некоторых гипотез эконометрического характера.

\section{Библиография :}

1. Банди, Б. Методы оптимизации. Вводный курс / Б. Банди. - М.: Радио и связь, 1988. - 128 с.

2. Бородин, А. В. Игры на сетях Петри / А. В. Бородин // Обозрение прикладной и промышленной математики. - 2002. - T. 9. - B. 1. - C. 167-168.

3. Бородин, А. В. Математические модели управления кредитным портфелем коммерческого банка / А. В. Бородин. - Йошкар-0ла: Марийский государственный технический университет, 1998. - 168 с.

4. Бородин, А. В. Моделиуправленияперсоналом в розничной подсистеме коммерческого банка / А. В.Бородин // Экономика и социум: современные модели развития общества в аспекте глобализации: материалы III международной научно-практической конференции (12 февраля 2014г.). - Саратов: Издательство ЦПМ «Академия Бизнеса», 2014. - С. 26-30.

5. Бородин, А. В. Сети Петри с нечетким поведением в задачах имитационного моделирования эволюции инвестиционных и страховых портфелей / А. В. Бородин // Обозрение прикладной и промышленной математики. - 2000. - Т. 7. - В. 2. - С. 321-322.

6. Бородин, А. В. Теоретико-игровые модели процессов риска над сетями Петри / А. В. Бородин // Моделирование и анализ безопасности и риска в сложных системах: Труды международной научной школы МАБР-2006. - СПб.: ГОУ ВПО «СПбГУАП», 2006. - С. 305-307.

7. Бородин, А. В. Управление HR-процессом в коммерческом банке на основе технологий имитационного моделирования / А. В. Бородин // Технические науки - от теории кпрактике. Сборник статей по материалам XXXII международной научно-практической конференции. № 3 (28). - Новосибирск: Издательство «СибАК», 2014. - C. 7-13.

8. Грант, К. Л. Управление рисками в трейдинге: Как повысить прибыльность с помощью контроля над рисками / К. Л. Грант. - М.: Мир, 2005. - 349 с.

9. Дамодаран, А. Инвестиционная оценка. Инструменты и техника оценки любых активов / А. Дамодаран. М.: Альпина Бизнес Букс, 2004. - 1342 с.

10. Джекел, П. Применение методов Монте-Карло в финансах / П. Джекел. - М.: Интернет-трейдинг, 2004 - 263 с.

11. Дубров, А. М. Моделирование рисковых ситуаций в экономике и бизнесе / А. М. Дубров, Б. Н. Лагоша, Е. Ю. Хрусталев. - М.: Финансы и статистика, 2003. - 223 с. 
12. Ермаков, С. М. Метод Монте-Карло в вычислительной математике. Вводный курс / С. М. Ермаков. - СПб.: Невский Диалект, Бином. Лаборатория знаний, 2009. - 192 с.

13. Уразаева, Т. А. Алгебраические аспекты имитационного моделирования портфелей срочных финансовых инструментов / Т. А. Уразаева, А. В. Бородин // Материалы конференции «Имитационное моделирование. Теория и практика». ИММОД-2013. Т. 1. - Казань: Издательство «Фэн» Академии наук РТ, 2013. - С. 282-286.

14. Уразаева, Т. А. Алгебра рисков / Т. А. Уразаева. - Йошкар-Ола:Поволжский государственный технологический университет, 2013. -209 c.

15. Arora, S. Computational Complexity: A Modern Approach / S. Arora, B. Barak. - New York: Cambridge University Press, 2009. $-579 p$.

16. Holton, G. A. Value-at-Risk: Theory and Practice / G. A. Holton. - Academic Press, 2003. - 405 p.

17. Papadimitriou, C. H. Computational complexity / C. H. Papadimitriou. - New York: Addison-Wesley Publishing Company, Inc., 1994. - 523 p.

18. Sipser, M. Introduction to the Theory of Computation / M. Sipser. - Boston: Thomson Course Technology, 2006. $-431 p$.

19. Чхутиашвили Л.В. Российские коммерческие банки в современных условиях: основные направления качественного совершенствования и перспективы развития // NB: Финансовое право и управление. -

2013. - 2. - C. 51 - 76. D0I: 10.7256/2306-4234.2013.2.641.URL: http://www.e-notabene.ru/flc/article_641.html

20. Анисимова 3.М. Повышение эффективности стратегического банковского менеджмента в современных условиях // Тренды и управление. - 2013. - 4. - С. 83 - 102. D0I: 10.7256/2307-9118.2013.4.10522.

21. В.Л. Шульц Сценарный анализ в управлении социальной безопасностью // Национальная безопасность / nota bene. - 2012. - 6. - C. 4 - 21.

\section{References:}

1. Bandi, B. Metody optimizatsii. Vvodnyi kurs / B. Bandi. - M.: Radio i svyaz', 1988. - 128 s.

2. Borodin, A. V. Igry na setyakh Petri / A. V. Borodin // Obozrenie prikladnoi i promyshlennoi matematiki. - 2002. -T. 9. - V. 1. - S. 167-168.

3. Borodin, A. V. Matematicheskie modeli upravleniya kreditnym portfelem kommercheskogo banka / A. V. Borodin. loshkar-Ola: Mariiskii gosudarstvennyi tekhnicheskii universitet, 1998. - $168 \mathrm{~s}$.

4. Borodin, A. V. Modeli upravleniya personalom v roznichnoi podsisteme kommercheskogo banka / A. V. Borodin // Ekonomika i sotsium: sovremennye modeli razvitiya obshchestva v aspekte globalizatsii: materialy III mezhdunarodnoi nauchno-prakticheskoi konferentsii (12 fevralya 2014g.). - Saratov: Izdatel'stvo TsPM «Akademiya Biznesa», 2014. - S. 26-30.

5. Borodin, A. V. Seti Petri s nechetkim povedeniem v zadachakh imitatsionnogo modelirovaniya evolyutsii investitsionnykh i strakhovykh portfelei / A. V. Borodin // Obozrenie prikladnoi i promyshlennoi matematiki. -2000. - T. 7. - V. 2. - S. 321-322.

6. Borodin, A. V. Teoretiko-igrovye modeli protsessov riska nad setyami Petri / A. V. Borodin // Modelirovanie i analiz bezopasnosti i riska v slozhnykh sistemakh: Trudy mezhdunarodnoi nauchnoi shkoly MABR-2006. - SPb.: GOU VPO «SPbGUAP», 2006. - S. 305-307. 
7. Borodin, A. V. Upravlenie HR-protsessom v kommercheskom banke na osnove tekhnologii imitatsionnogo modelirovaniya / A. V. Borodin // Tekhnicheskie nauki — ot teorii k praktike. Sbornik statei po materialam XXXII mezhdunarodnoi nauchno-prakticheskoi konferentsii. № 3 (28). - Novosibirsk: Izdatel'stvo «SibAK», 2014. - S. 7-13.

8. Grant, K. L. Upravlenie riskami v treidinge: Kak povysit' pribyl'nost's pomoshch'yu kontrolya nad riskami / K. L. Grant. - M.: Mir, 2005. - 349 s.

9. Damodaran, A. Investitsionnaya otsenka. Instrumenty i tekhnika otsenki lyubykh aktivov / A. Damodaran. - M.: Al'pina Biznes Buks, 2004. - 1342 s.

10. Dzhekel, P. Primenenie metodov Monte-Karlo v finansakh / P. Dzhekel. - M.: Internet-treiding, $2004-263 \mathrm{~s}$.

11. Dubrov, A. M. Modelirovanie riskovykh situatsii v ekonomike i biznese / A. M. Dubrov, B. N. Lagosha, E. Yu. Khrustalev. - M.: Finansy i statistika, 2003. -223 s.

12. Ermakov, S. M. Metod Monte-Karlo v vychislitel'noi matematike. Vvodnyi kurs / S. M. Ermakov. - SPb.: Nevskii Dialekt, Binom. Laboratoriya znanii, 2009. - $192 \mathrm{~s}$.

13. Urazaeva, T. A. Algebraicheskie aspekty imitatsionnogo modelirovaniya portfelei srochnykh finansovykh instrumentov / T. A. Urazaeva, A. V. Borodin // Materialy konferentsii «Imitatsionnoe modelirovanie. Teoriya i praktika». IMMOD-2013. T. 1. - Kazan': Izdatel'stvo «Fen» Akademii nauk RT, 2013. - S. 282-286.

14. Urazaeva, T. A. Algebra riskov / T. A. Urazaeva. - loshkar-0la: Povolzhskii gosudarstvennyi tekhnologicheskii universitet, 2013. $-209 \mathrm{~s}$.

15. Arora, S. Computational Complexity: A Modern Approach / S. Arora, V. Barak. - New York: Cambridge University Press, 2009. $-579 p$.

16. Holton, G. A. Value-at-Risk: Theory and Practice / G. A. Holton. - Academic Press, 2003. - 405 p.

17. Papadimitriou, C. H. Computational complexity / C. H. Papadimitriou. - New York: Addison-Wesley Publishing Company, Inc., 1994. -523 p.

18. Sipser, M. Introduction to the Theory of Computation / M. Sipser. - Boston: Thomson Course Technology, 2006. $-431 p$.

19. Chkhutiashvili L.V. Rossiiskie kommercheskie banki v sovremennykh usloviyakh: osnovnye napravleniya kachestvennogo sovershenstvovaniya i perspektivy razvitiya // NB: Finansovoe pravo i upravlenie. - 2013. - 2. - C. 51 - 76. D0I: 10.7256/2306-4234.2013.2.641.URL: http://www.e-notabene.ru/flc/article_641.html

20. Anisimova Z.M. Povyshenie effektivnosti strategicheskogo bankovskogo menedzhmenta v sovremennykh usloviyakh // Trendy i upravlenie. - 2013. - 4. - C. 83 - 102. D0l: 10.7256/2307-9118.2013.4.10522.

21. V.L. Shul'ts Stsenarnyi analiz v upravlenii sotsial'noi bezopasnost'yu // Natsional'naya bezopasnost' / nota bene. 2012. - 6. - C. 4 - 21. 